\title{
El pintor don Diego de Silva que no es Veláz- quez
}

\author{
José Manuel CRUZ VALDOVINOS \\ Universidad Complutense de Madrid
}

\begin{abstract}
RESUMEN. Diego de Silva fue un pintor cuyas noticias se han atribuido en ocasiones a Velázquez por haber coincidido su estancia en Roma en 1630 con la del sevillano y cuya presencia en la demarcación de San Lorenzo in Lucina en 1636 dio origen a la ya desechada teoría de un viaje de Velázquez a Roma en ese año. Pero el interés sobre su figura radica principalmente en haber disfrutado de una licencia de Felipe IV para realizar en El Escorial copias de las pinturas que allí había, en especial de los tizianos, a partir de 1642 o 1643 en que se trasladó a España. Ejerció también como teniente de conserje del Escorial entre 1668 y 1690 en que murió. Aportamos diversas noticias profesionales y familiares, en especial sobre su hijo, el también pintor y teniente de conserje del Escorial, Felipe de Silva.

Palabras clave: Diego de Silva, Felipe de Silva, Velázquez, Herrera Barnuevo, El Escorial, Tiziano, conserje, Pötting.

ABSTRACT. Diego de Silva was a painter who has been sometimes mistaken on some news for Velázquez due to the fact that both coincided during their time in Rome in 1630 and that their presence at Saint Lorenzo in Lucina's district in 1636 gave raise to the already rejected theory that Velázquez travelled to Rome at that year. But the interest on this man lies mainly in the licence that Phillip IV granted him to make copies of the paintings in El Escorial, especially those of Tiziano, from 1642 or 1643, when he came to Spain. He also worked as deputy concierge in El Escorial between 1668 and 1690, when he died. We provide some formal and informal news, especially related to his son, also painter and deputy concierge of El Escorial, Phillip de Silva.

Key words: Diego de Silva, Felipe de Silva, Velázquez, Herrera Barnuevo, El Escorial, Tiziano, concierge, Pötting.
\end{abstract}

En 1942, Hoogewerff (1884-1963), ilustre investigador de la pintura holandesa del siglo XVII, publicó un extenso volumen con noticias sobre artistas holandeses extraidas de archivos parroquiales romanos ${ }^{1}$. En ese libro, correspondiendo al año 1636 y

\section{-}

${ }^{1}$ G. J. HOOGEWERFF, Nederlandsche Kunstenaarste Rome (1600-1725). Uittreksels uit de Parrochiale Archieven, La Haya, 1942. procedente de la Descrittione dell'anime della parocchia di S. Lorenzo in Lucina, folio 13, se anota: "Borgognani dal Corso, mano sinistra, Francesco de Lara, pittore c. Michele Medrano/ (Als vorachtig in dezelfde straat komt in dit jarr a.o.) Diego de Sylva Velazquez". La noticia apenas alcanzó difusión hasta que Enriqueta Harris dio cuenta de ella en un artículo de 1958. La autora había revisado el padrón del cumplimiento de la comunión por Pascua Flori- 
da o Resurrección de dicha parroquia encontrando a Lara y Medrano, pero no "la referencia a Velázquez ni ningún nombre semejante" ${ }^{\prime 2}$. Aunque solicitó más información al doctor holandés, éste no pudo añadir otra cosa en vista del tiempo transcurrido desde que, durante el inicio de la segunda Guerra Mundial, había realizado su investigación en Roma y quizá también, pensamos, por su avanzada edad.

Con motivo de la celebración del III centenario de la muerte del pintor sevillano, tuvo lugar una gran exposición en el Casón del Buen Retiro de Madrid y se publicaron dos volúmenes con estudios, textos referidos al pintor y recopilación de los más de doscientos documentos que hasta esa fecha se habían dado a conocer sobre Velázquez. Entre ellos se recogió la noticia transcrita de Hoogewerff titulando el documento: "¿Noticia de una posible presencia de Velázquez en Roma en 1636?, con una nota comentando lo expuesto por Harris en que, alineándose con la estudiosa, se afirmaba que el viaje no se debió de realizar. Pero también se advertía que don Gaspar de Fuensalida, uno de los testigos en el expediente informativo para la concesión del hábito de la orden de Santiago al sevillano, había declarado que éste fue tres veces a Italia ${ }^{3}$.

En el seminario "Reflexión sobre Velázquez", celebrado en agosto de 1990 dentro de los cursos de verano de la Universidad Complutense de Madrid, el profesor Pita Andrade volvió sobre el "posible y probable" viaje de 1636 a Italia. Su pensamiento lo desarrolló y publicó poco des-

\footnotetext{
2 E. HARRIS, «Velázquez en Roma», Archivo Español de Arte, 123, 1958, pp. 185-192, en especial 185, n. 2.

${ }^{3}$ Varia Velazqueña, Madrid (Ministerio de Educación Nacional) 1960, II, pp. 242-243, nº 67.
}

pués ${ }^{4}$. Aún reconociendo que Harris no había encontrado la noticia publicada por Hoogewerff que era el fundamento de la argumentación, defendía su veracidad y, por tanto, la estancia de Velázquez en Roma en 1636. Su especulación le conducía a justificar el viaje para acompañar el modelo de escultura del rey que había hecho Montañés y el retrato ecuestre pintado por el propio Velázquez, a fin de que Pietro Tacca los usara para su estatua de bronce del monarca, según escribieron Ponz en 1772 y Ceán en 1800. La ayuda de costa recibida por el sevillano en 1637 de que hablaba Palomino se relacionó por Pita con los gastos del viaje, a pesar de que fue satisfecha a posteriori. Velázquez habría estado en Italia casi todo el año 1636 y buena parte de 1637, y volvería al final de éste para participar en la decoración de la Torre de la Parada. Recoge la noticia de que la junta de Obras y Bosques acordó el 24 de octubre de 1636 consultar al rey sobre el memorial presentado por el sevillano en que menciona que se le ha mandado pintar para la Torre, pero no parece que fuera consciente de la dificultad que originaba para su teoría, en razón de la fecha. Admitió como obstáculo que ni Pacheco ni Jusepe Martínez ni Lázaro Díaz del Valle ni Palomino hicieran mención alguna al supuesto viaje, pero dio más importancia a las declaraciones de Fuensalida -ya mencionadas- y de Muñoz Gamboa, contralor de la casa de la Reina, y del marqués de Malpica, mayordomo del Rey, hechas en la misma ocasión, quienes dijeron que Velázquez había ido muchas veces a Italia, y no tuvo en cuenta la vaguedad e imprecisión de estas afirmaciones como tantas otras en el expediente ${ }^{5}$. La honradez

${ }^{4}$ J. M. PITA ANDRADE, Del Retiro a la Torre de la Parada ipasando por Italia?: el posible viaje de 1636 en Velázquez y el arte de su tiempo. V Jornadas de Arte del C.S.I.C, Madrid (Alpuerto) 1991, pp. 119-126.

5 Todavía añadió una afirmación, discutible a nuestro juicio, de que nadie dudaba que en el Marte se 
científica que caracterizaba al profesor Pita Andrade le llevó a rectificar años después ${ }^{6}$, tras conocer la publicación de Fernando Marías a la que nos referimos a continuación.

Marías examinó el libro romano sobre cumplimientos pascuales ya citado, que se conserva en el Archivio Storico del Vicariato di Roma (Stati delle Anime, 20, San Lorenzo in Lucina 10. Stati d'Anime, 1636). La transcripción literal que presenta el autor ${ }^{7}$ demuestra, en primer lugar, que Hoogewerff sólo copió algunos nombres de toda la relación, pero, sobre todo, que el llamado don Diego de Silva vivía en calle diferente a la de Lara y Medrano y no aparecía nunca citado con un segundo apellido. Aunque pueda resultar una lista algo larga, la copiaremos aquí para comodidad y mejor comprensión del lector: En la via Borgognoni dal Corso mano sinistra: "Giovanni palafrenero del Sr. Ambaciatore di Spagna/ Giovanni Batista Villamaior del Sr. Ambaciatore di Spagna/ Claudio cocciero del Sr. Ambaciatore di Spagna/ Nicolo garzon di stalla del Sr. Ambaciatore di Spagna/ Sr. D. Josepe di Sosa/ [tachado] Agostino Nerocchi pittore/D. Michele Medrano gentilhuomo del Imbaciatore (sic)/D. Francesco de Cespedes/ Francesco de Lara pittore" (fol. 12v-13v). Algunos folios después, en la via della Sirena can.do della Trinitá mano destra: "Francesco

fundían recuerdos del Pensieroso de Miguel Ángel y del Ares Ludovisi y que concibió Velázquez la obra partiendo de la impresión directa que le produjeron ambas esculturas, y que la primera hubo de verla en Florencia en este supuesto viaje, puesto que no estuvo en la ciudad en el primero de 1629-1631.

${ }^{6}$ J. M. PITA ANDRADE, José Manuel, «Interrogantes sobre Velázquez en sus etapas madrileñas», Madrid. Revista de Arte, Geografía e Historia 2, 1999, pp. 328-329. Confiesa que pecó de ingenuo al fiarse del rigor científico de Hoogewerff.

${ }^{7}$ F. MARÍAS, «Sobre el número de viajes de Velázquez a Italia», Archivo Español de Arte, 258, 1992, pp. 218-221.
Guetier pittore/ Giovanni Ghinetto cocchiero/ Domenico Innocentio cocchiero/ D. Diego de Sylva no c./ D. Gaspar Pesció no c./ Gioseppe Ottengha no c./ D. Luca Cannoniero/ Pietro Marocchi muratore/ Giov. Battista Lucchese pittore/ D.Michele Planella" (fol.18 v).

El citado investigador encontró que en el libro correspondiente a 1637 y, en la misma via della Sirena -aunque dice a mano izquierda, pero quizá la anotación se hizo yendo de la Trinitá al Corso y no al contrario como el año precedente- figuran: “D. Luca sacerdote c./ D.Diegho di Silva c./ Francesco servitore c." (sin foliar). Y aún señala Marías que en el último libro conservado de estos años -si bien no precisa cuáles incluye- que es de 1634, "via della Sirena a mano sinistra", aparecen: "D. Alvaro de Almeyda c./ D.Giovanni Paniagua c./ D.Gregorio Ramirez c./ D.Diego de Sylva c." (fol. $23 \mathrm{v}$ ).

Hay que destacar que se trata de una demarcación de la parroquia de San Lorenzo in Lucina que, cruzando la via del Corso, se extendía hacia la Trinitá y el palacio de España (todavía conserva el nombre la via Borgognona), una zona donde habitaban criados del embajador español, entonces don Manuel de Moura marqués de Castelrodrigo, y también bastantes castellanos y portugueses. Por ello y porque el apellido Silva era bastante común en Castilla y en Portugal no cabe afirmar cuál era la nación del personaje que nos ocupa, que, a diferencia de alguno de los citados, no es mencionado como pintor y que, desde luego, no lleva el apellido Velázquez ${ }^{8}$.

\footnotetext{
${ }^{8}$ El holandés añadió por su cuenta el apellido Velázquez. Quizá hizo una anotación en la papeleta sugiriendo que pudiera ser Velázquez y luego la incorporó a la publicación olvidando su carácter de sugerencia.
} 
No merece la pena enumerar los muchos documentos firmados en Madrid en 1636 y 1637 por el pintor Velázquez o las noticias de las que se deduce su presencia en la corte de Felipe IV en ese tiempo. Nos remitimos a los recogidos en el Corpvs velazqueño ${ }^{9}$. Hace tiempo, por tanto, que quedó resuelta de forma negativa la pregunta que planteaba la publicación antológica de 1960. Velázquez no viajó a Italia en 1636 ni en otros momentos que no fueran los conocidos de 1629-1631 y 1648-1651.

El objeto de este trabajo es seguir el rastro del don Diego de Silva que estaba en Roma en 1634-1637, que no era Velázquez pero sí pintor, y a quien se pueden referir numerosas noticias anteriores y posteriores a dicho periodo.

En 1996 se publicaron documentos sobre un "don Diego" pintor en Roma ${ }^{10}$. El 6 de enero de 1630 "Don. Diego pitor dono per pigione di tre mesi cominciati a 6 gennaro, scudi 13,75". El cuarto arrendado por este pintor estaba en la casa Mannara en via Paolina. Los pagos por el alquiler se repiten el 9 de agosto por los seis meses desde 6 de abril hasta el 6 de octubre, satisfaciendo 27,50 escudos, el 25 de septiembre por otros tres meses hasta el 6 de enero de 1631 y el 20 de abril por tres meses más, hasta el 6 de julio, siempre por importe de 13,75 escudos por trimestre.

Poco después, Salort señalaba que "no se sabe con certeza si se trata de Velázquez"11 y Morán Turina duda en 2008 de

\footnotetext{
${ }^{9}$ Corpus velazqueño, Madrid, 2000, I, pp. 112-126.

${ }^{10}$ G. MicheL, «Nicolas Poussin et la maison Mannara», Gazette des Beaux Arts 127, 1996, pp. 213-220. Los documentos en Archivio della Confraternità dei Bergamaschi. Libro maestro B, fol, 196. Las noticias, sin observación alguna, se recogieron en Corpvs cit., doc 72 a.

11 S. SAlORT PONS, Velázquez en Italia, Madrid, 2002, p. 400.
}

que sea el sevillano, pues el último pago registrado, de 20 de abril de 1631, "resulta difícil de conciliar con el hecho de que abandonara la ciudad en noviembre de 1630 para marchar a Nápoles y desde allí regresar a España, donde llegó a principios del año siguiente"12. No sólo es difícil sino imposible que se tratara de Velázquez, pues el contrato se prorrogó más de siete meses después de que abandonara Roma y, por otra parte, sabemos que vivió en el Palacio Vaticano primero, en la villa Medicis después -la solicitud del embajador español para que le permitieran habitar en este palacio fue posterior al arrendamiento y coincide aproximadamente con la prórroga primera, de abril de 1630- y, por último, tras haber enfermado a fines del verano de 1630, se trasladó a las inmediaciones de la embajada de España, siempre alojado de forma gratuita.

Aunque en las noticias de 1630-1631 no se menciona que el pintor se apellidara Silva y en las de 1634-1637 no consta que fuera pintor, no es mucho atrevimiento pensar que se trataba de la misma persona, pues hay elementos suficientes que lo apoyan. El alquiler en casa Mannara le relaciona con el pintor normando Nicolas Poussin, que vivía desde 1626 allí -hasta 1631 no consta que se trasladara a la via del Babuino y precisamente cesa en julio de 1631 la noticia documentada sobre el arrendamiento del pintor Diego- y con el escultor bruselés François Duquesnoy, quien en 1636, aparece también en el status animarum de San Lorenzo in Lucina, pues vivía muy cerca de Silva ${ }^{13}$. Esta relación con artífices de habla francesa será un hecho importante para identificarle como el pintor afincado

\footnotetext{
12 A. Palomino, Vida de don Diego Velázquez de Silva, ed.de Miguel Morán Turina, Madrid, 2008, p. 90, n.197.

13 A. MERot, Nicolas Poussin, París 1990, cronología; MICHEL cit., y MARÍAS cit.,
} 
en El Escorial del que nos ocupamos seguidamente.

El primer documento conocido que muestra al pintor don Diego de Silva como residente en la villa y corte de Madrid corresponde a 1653, pero, en realidad, estaba en España ya en 1643. Mediante escritura de 28 de mayo de este último año, otorgada en Plasencia, don Cristóbal López Floriano, caballero de la orden toscana de San Esteban y alcaide de la fortaleza real de dicha ciudad, le hizo cesionario de 4.780 reales para que los cobrase de la casa de los Fúcares de un principal de 1.466 .598 maravedís de vellón y de otro de 97.410 maravedís de plata doble que tenía en depósitos en esa banca desde enero de 1634. Como Silva no había conseguido cobrarlos por la quiebra de los Fúcares, la viuda del cedente, doña Antonia Sardeneta, se convino con él diez años después para que le hiciera retrocesión de sus derechos contra esa casa, dándole 3.000 reales de vellón. Los testamentarios de la viuda se obligaron a pagar tal cantidad al pintor, a plazos y éste hizo la retrocesión mediante escritura otorgada en Madrid a 6 de noviembre de $1653^{14}$.

La noticia de que, desde 1643 o 1644, un don Diego de Silva pintor estaba empleado en el real sitio del Escorial con licencia de Felipe IV para realizar copias de Tiziano y otros surge a partir de un memorial presentado por él a fines de $1669^{15}$ para solicitar puesto de conserje de aquel sitio. Declara como mérito que llevaba 26 años en dicha ocupación de copista y que durante los dos últimos había ejercido como teniente de la plaza solicitada. Alegaba también que su padre había sido pagador de basti-

\footnotetext{
14 M. AgUlló y COBO, Documentos para la Historia de la pintura española III, Madrid, 2006, p. 269.

15 Archivo General de Palacio (A.G.P.), expediente personal, 507/40.
}

mentos del castillo de Amberes durante 29 años.

El documento clave para revelar que las anteriores noticias corresponden a la misma persona se data en 1660, con ocasión de sus desposorios. El 20 de enero de dicho año "don Diego de Silva, maestro pintor del real sitio del Excurial" 16 -obsérvese el uso del "don" y su profesión- declaró que había conferido velarse con doña Mariana de Grijalva y Riaño, hija legítima de Eugenio Llorente de Grijalva y de doña Clara de Vitoria y Riaño ${ }^{17}$. Añade que "por las muchas obras y ocupaciones que tiene en el ministerio del dicho su oficio y ser considerables y de tanta importancia, no puede ausentarse del dicho real sitio ni acudir para las diligencias y concierto", por lo que había otorgado poder a don Lorenzo de Soto, maestro pintor vecino de Madrid. Le autorizó a concertar la dote que habían de

${ }^{16}$ Debemos el conocimiento de la escritura a Juan María Cruz Yábar, conservador del Departamento de Edad Moderna en el Museo Arqueológico Nacional de Madrid. Se halla en el Archivo Histórico de Protocolos de Madrid (A.H.P.M.), prot. 8958, fol 359-359v, Documento $\mathrm{n}^{\mathrm{o}} \mathbf{1}$.

17 J. M. CRUZ VALDOVINOS, La platería y los plateros de Madrid desde 1624 hasta 1695, Pamplona 1968 (tesis de licenciatura inédita). Llorente era platero, a quien tenemos documentado desde 1617 a 1649. El 1 de julio de 1617 ingresó en la hermandad de mancebos plateros de San Eloy de Madrid, por lo que nacería a comienzos del siglo XVII, y el 20 de marzo de 1627 fue aprobado por la congregación de San Eloy como maestro platero de oro, y por ese tiempo pudo contraer matrimonio. El 30 de junio siguiente figura con diez reales en el repartimiento que se hizo entre los plateros por el servicio del $1 \%$ correspondiente al primer medio año de 1627. Para el gasto del adorno que hicieron los plateros en 1649 en el recibimientode la reina Mariana contribuyó con 20 reales. C. PÉREZ PASTOR, Documentos para la bibliografía de don Pedro Calderón de la Barca, Madrid, 1905, pp. 131-133; A.H.P.M.,escr. Juan de Burgos, da cuenta de que el 16 de mayo de 1646 fue testigo en la información sobre la muerte acaecida en 1645 de José Calderón de la Barca, a quien dijo conocer desde hacía veinte años, a petición de sus hermanos Diego y Pedro, por motivo de la herencia. 
dar los padres de doña Mariana, ofrecer las arras y hacer las capitulaciones. Debía de tratarse del segundo matrimonio del pintor, pues consta que tenía hijos nacidos antes de esta fecha.

Muy cercana es la siguiente noticia, de 15 de febrero de 1660, en que el pintor Antonio Ponce (1608-1677) recibió el dinero de un anillo de diamantes que Diego de Herrera Barnuevo había vendido a un Diego de Silva, que ha de ser el que nos ocu$\mathrm{pa}^{18}$. Herrera había sido aprobado como maestro platero de oro por la congregación de San Eloy de artífices plateros de Madrid el 27 de febrero de 1638 y fue mozo del oficio de guardajoyas del Rey; elegido mayordomo de la Congregación en 1658, pasó a ser diputado al año siguiente, pero falleció en noviembre antes de cumplir el año reglamentario. Nombró como albacea a Ponce, que estaba casado con Francisca de Alfaro, hija de Juana de Herrera, hermana de Diego, y del platero francés Jean Jacques de las Nieves, aprobado también en la especialidad de oro el mismo día que Herre$\mathrm{ra}^{19}$. La relación entre Diego de Silva y los Herreras Barnuevos pudo originarse a partir de la que tendría con Jean Jacques, ya que el pintor conocería bien el idioma francés si había vivido mucho tiempo en Amberes.

En 1669, don Diego de Silva hizo una copia de un cuadro de Tiziano del monasterio del Escorial por encargo del conde de Pötting, embajador imperial en Madrid ${ }^{20}$. El martes 30 de julio de ese año, Pötting anotó en su Diario: "Pagué por un lienço de una copia de San Lorenço de aquel famosso

\footnotetext{
18 A.H.P.M., prot 9147, fol.145.

${ }^{19}$ J. M. CRUZ VALDOVINOS cit, ad nomines.

${ }^{20}$ F. E. von PötTING, Diario del conde de Pötting, embajador del Sacro Imperio en Madrid (1664-1674), ed. de Miguel Nieto Nuño, Madrid, 1990 (tomo I) y 1993 (tomo II), ad datam todas las noticias que citamos.
}

original de Ticiano en Escorial, 200 pesos, que lo vale muy bien. Híçole don Diego de Sylva, pintor del rey de aquel sitio". El título de pintor real que utiliza Pötting es una mención convencional que no se debe entender en sentido estricto, pues el propio Silva describirá con exactitud en el memorial que hará a fines de ese mismo año 1669 la calidad de su empleo en el Monasterio, que era la de una licencia real para poder hacer copias. La cantidad pagada por el embajador equivalía a 3.000 reales de vellón. La obra de Tiziano fue pintada para Felipe II en 1566-1567 aunque el encargo se hizo a finales de $1564^{21}$. El lienzo mide $418 \mathrm{x}$ $297 \mathrm{~cm}$ y hay que suponer que la copia de Silva sería de tamaño reducido, pero el número de figuras -once, más dos angelitos, además de un caballo, perro y estatua- justifica el precio, que el embajador consideró moderado. Conviene tener en cuenta que el conde de Pötting había nacido el 10 de agosto de 1627, festividad del diácono san Lorenzo, por quien consta que tenía especial devoción como se deduce del texto de su Diario.

Dos meses más tarde, el 29 de septiembre, el pintor entregó al embajador otra copia de Tiziano: "El pintor don Diego de Sylva del Escorial me presentó una muy buena copia de la Madalena penitente de Tyziano" 22 . El veneciano envió el original a Felipe II en 1561; no se conserva y es conocido por diversas copias, pero no podemos identificar entre ellas la de Silva ${ }^{23}$. En esta

${ }^{21}$ C. GARCÍA-FríAs CHECA y E. RODRÍGUEZ-ARANA MUÑOz, Tiziano y el Martirio de San Lorenzo en El Escorial, Madrid, 2003.

${ }^{22}$ Las noticias de julio y septiembre fueron recogidas por Bassegoda, quien consideró a Silva antepasado de Felipe y Diego, a lo que luego nos referimos (B. BASSEGODA I HUGAS, El Escorial como Museo, Barcelona, 2003, 59).

${ }^{23}$ P. Beroqui, Ticiano en el Museo del Prado, Madrid 1946; F. VALCANOVER, L'opera completa di Tiziano, Milán, 1969, no 433. 
ocasión no consta que hubiera encargo ni pago de Pötting y debemos entender que fue un obsequio del pintor, quizá relacionado con la noticia que se expone a continuación.

Al día siguiente, lunes 30 de septiembre de 1669, el embajador anotó: "Esta tarde entró a servir a la Condessa una doncella, doña Josefa de Sylva, hixa del pintor de San Lorenço. Tiene muy buena voç y canta bonicamente". Pero, antes de que transcurriera un año, el sábado 2 de agosto de 1670, de manera sorprendente, la condesa de Pötting despidió a su criada "por hauer ésta instado sobre ello disparatadamente" como escribe de forma hermética el embajador.

Entre tanto, había muerto Manuel de Herrera Barnuevo -hijo del que fue escultor real Antonio de Herrera- que ocupó la conserjería del Escorial desde 1666 a 1669. Su hermano, Sebastián de Herrera Barnuevo, pintor de cámara y maestro mayor de las obras reales, solicitó la plaza y el memorial se remitió a la Reina el 12 de diciembre de 1669; del mismo resulta que el fallecido ejercía a la vez en Madrid el oficio de ayuda de la furriera, tal y como había hecho su predecesor, Joaquín Cobos. Puesto que la presencia en el Escorial del conserje sólo era necesaria cuando el rey estaba allí, se le había concedido la facultad de que tuviera un teniente que residiera en el real sitio, para lo que debió designar a Diego de Silva. En fechas cercanas solicitaron también la plaza el pintor Francisco Ricci, Toribio González de los Corrales regidor de Oviedo, Mateo Díaz de Langarica y Mendoza agente de los presidios de Guipúzcoa, Juan Delgado ayuda de la tapicería, Francisco Patiño ayuda de la cava, Juan López Monje y Carlos de Salazar mozos de la furriera, Gaspar de la Cuesta escudero de a pie y el propio Diego de Silva con el memorial que hemos citado anteriormente. Se elevaron las peticiones a consulta de la junta de Obras y
Bosques el 2 de enero de 1670, que entregó a la reina Mariana su informe el 15 de enero. Además de dar cuenta de que la plaza tenía 400 ducados anuales de gajes, de los que 100 se daban como pensión a una hija de Joaquín Cobos, la Junta proponía en primer lugar a Sebastián de Herrera, en segundo a Toribio González y en tercero a Diego de Silva. Sobre este último señalan que había sido el interpuesto de Herrera Barnuevo, que residía en Madrid la mayor parte del año, y que, además de tener en orden el aposento real, se había ocupado "asimismo en lo que en el discursso deste tiempo se ha ofrecido en el exercicio de pintor, lo que ha sido requerido en aquel palacio real y combento" ${ }^{24}$. En su memorial, Silva mencionaba su relación con Amberes -que ya citamos-, los 26 años que asistió en el real sitio "con su familia", y que estuvo empleado "con el arte de la pintura en copiar originales del Tiçiano y otros", de lo que es ejemplo las noticias de Pötting; afirmaba también que los dos "postreros años" había servido con el oficio de teniente de conserje de aquella real casa ${ }^{25}$, lo que hace coincidir el comienzo de sus funciones de conserje con el tiempo en que fue titulár Manuel de Herrera. La Reina eligió para la plaza a Sebastián de Herrera Barnuevo, que ocupó el cargo hasta su muerte en 1671, y fue sucedido por su hijo Ignacio hasta 1708. Sin duda, Silva siguió ejerciendo su tenencia de conserje hasta su muerte $\mathrm{y}$, a su vez, le sucedió en ella su hijo, tal como revela otro memorial del que nos ocupamos más adelante. No debía ser una plaza de plantilla -al menos hasta varios años después de la llegada de los Borbones-, de modo que sería retribuída por los sucesivos titulares, de quienes dependía el nombramiento del teniente. Sebastián de Herrera debía tener interés en esa plaza para conservarla para

\footnotetext{
${ }^{24}$ Documento no 2.

${ }^{25}$ Ibidem.
} 
su familia, pues poco le debía quedar de los 300 ducados después de pagar a su teniente.

Diego de Silva tenía a su cargo, a comienzos de 1670, mujer y cinco hijos, como declara en el memorial. Sólo tenemos noticias de tres de esos hijos. Josefa y Felipe, que consideramos anteriores a su matrimonio con Mariana en 1660, intentaron acomodarse cuando llegaron a una edad apropiada para ello. Ya hemos mencionado el episodio de Josefa, y nada más sabemos de ella. Felipe fue también pintor y poseemos algunas noticias suyas. En 1689 declaró tener unos 36 años, por lo que nacería hacia 1653. Un tercer hijo de Diego de Silva llamado Manuel -seguramente del segundo matrimonio-, solicitó en 1694 el ingreso en la guardia de archeros de $\operatorname{corps}^{26}$, en la que no parece que llegara a ser admitido. Las plazas de archero eran conferidas habitualmente a flamencos, e invocaría para ello las raíces antuerpienses de su padre y abuelo.

El 6 de febrero de 1670, Alonso del Arco concertó enseñar el arte de pintor a Antonio Guerrero por mil reales; Felipe de Silva es uno de los tres testigos del documento ${ }^{27}$. Podría quedar planteada la hipótesis de que Silva fuera oficial de Alonso del Arco, pues tendría ya 17 o 18 años y habría aprendido con su padre.

Transcurrido un tiempo, Felipe de Silva está documentado en Madrid realizando tasaciones de pinturas. El 18 de agosto de 1677 las de Francisco García, oficial de sillero 28 ; el 20 de agosto de 1680 las de Ber-

\footnotetext{
${ }^{26}$ F. NAVArRo, C. MORTERero y G. PORRAs, Noble guardia de arqueros de Corps, Madrid, 1962, p. 149.

${ }^{27}$ M. AgUlló y COBO, Más noticias sobre pintores madrileños de los siglos XVI al XVIII, Madrid, 1981, p. 14.

${ }^{28}$ EADEM, Documentos cit., pp. 269-270.
}

nabé de Gainza, secretario del rey en la junta de Aposento ${ }^{29}$, el 10 de enero de 1681 las del vallisoletano José de Lezana, maestro platero de oro aprobado en $\mathrm{Madrid}^{30}$, el 12 de enero de 1681 las de Francisco Fortuny, secretario del marqués de Tamarit ${ }^{31} \mathrm{y}$ el 28 de abril de 1681 las de Nicolás de Espinosa, que iba a casar con doña Francisca de la Cuadra, viuda de Manuel de la Mota, criado del rey ${ }^{32}$.

Tras la muerte de Carlos II, el alcalde mayor de la villa del Escorial nombró el 10 de diciembre de 1700 "tasador por lo que toca a pinturas a Don Phelipe de Silva, vecino del sitio"33. El mismo día juró el oficio y en los días siguientes se ocupó de la tasación: el 11 y el 12 de las pinturas, el 13 asistió también a las de tapices y alfombras y el 14 a la tasación de muebles.

En un memorial sin fecha ${ }^{34}$, Felipe de Silva se titula teniente de conserje del real sitio del Escorial y escribe que "ha más de 50 años que ha estado sirviendo su padre don Diego de Silba y el suplicante lo a continuado desde el año de mil seiscientos y noventa". No se conoce la fecha del memorial y los índices temporales que contiene tampoco son claros. Si el rey era aún Carlos II, habría que deducir que se estaba refiriendo al establecimiento de su padre Diego en el Escorial, lo que sucedió hacia 1643 ó 1644 en que debió adquirir la condición de copista del monasterio con licencia real.

${ }^{29}$ A ATERIDO, La pintura del último barroco en Madrid (1685-1726), Madrid, 2010 (tesis doctoral inédita), p. 898.

${ }^{30}$ A.H.P.M., prot. 8181, fols 28-30; cfr. J. M. CRUZ VALDOVINOS cit., ad nominem.

${ }^{31}$ A. ATERIDO cit, 898; A.H.P.M., prot. 13.210, fols 41-45.

${ }^{32}$ M. Agulló y COBO, Documentos cit., p. 270.

${ }^{33}$ Inventarios reales. Testamentaría del rey Carlos II, Madrid, 1985, III, pp. 91 y ss.

34 A.G.P., expediente personal, 1000/19. 
Más acertada nos parece la interpretación de que el memorial se estaba dirigiendo ya a Felipe V en una fecha en torno a 1708, en que murió Ignacio de Herrera Barnuevo, y los cincuenta años mencionados de servicios de su padre serían alegados con la certeza de que no constaría en el Bureo el momento en que se inició el servicio como teniente de Diego de Silva, pues era una plaza no de plantilla y constaría, en cambio, que asistía con asiduidad al real sitio desde bastante antes. También podría deducirse que Diego se jubiló o, más probablemente, murió en 1690 y que Felipe le sucedió de inmediato; la falta de noticias sobre este pintor en Madrid a partir de 1682 pudo deberse a su traslado al Escorial para ayudar a su anciano padre y garantizarse así la sucesión en su tenencia de conserje. En el memorial se menciona que Silva cuidaba de las llaves y atendía a la limpieza del real Palacio y de las casas de oficios. Pedía una ayuda de costa y limosna basándose en precedentes, y así exponía que en las jornadas de todos los reyes anteriores al real sitio habían dado siempre 20 doblones de a dos escudos de oro para ayuda de los gastos que se hacían y para la limpieza, y 8 doblones más por vía de limosna y que, habiendo servido al rey en toda la jornada que había hecho, no se le había ayudado con cosa alguna, hallándose "con cortos medios y crezida familia". No consta la respuesta, si la hubo.

Tampoco lleva fecha y se desconoce el resultado de otra petición ${ }^{35}$ en que se titula ayuda de conserje del real sitio. Parece algo posterior al memorial citado en el párrafo precedente. El título de ayuda significaría que la plaza se había incluido en la plantilla de la casa real, pues es una denominación habitual en el escalafón de los criados del monarca. Declara que, cuando

\footnotetext{
35 Ibidem.
}

el rey estuvo en el Escorial, le había dado diferentes memoriales suplicándole mil reales de ayuda de costa para la limpieza de patios y escaleras del palacio y que, habiendo favorecido a otros dependientes de dicho real sitio, a él no se le había concedido merced alguna, en contra de lo que siempre se había estilado; añadía que se hallaba pobre y con diez hijos pequeños a los que sustentar.

La última noticia que conocemos de Felipe de Silva es la hechura de un cuadro, todavía conservado en la colección del Escorial, que representa a Felipe $V$ defensor de la Fe contra la Herejía, que se data hacia 1712, pues fue encargado por los monjes jerónimos del monasterio para conmemorar la victoria en la batalla de Villaviciosa ${ }^{36}$.

En conclusión, consideramos que el don Diego pintor que aparece arrendando un cuarto en casa Mannara de Roma en enero de 1630 ha de ser el mismo don Diego de Silva que documentamos en Madrid a partir de 1643. El hecho de que se halle a este entonces joven pintor en el entorno de Poussin, que vivía en la citada casa romana, no resulta extraño, pues Silva hablaría francés como su lengua materna por haberse criado -y quizá nacido- en Amberes. Su padre, pagador de bastimentos del castillo de la ciudad, sería un hombre de negocios seguramente portugués a juzgar por su apellido y su oficio -los pagadores habían de adelantar habitualmente el dinero a la hacienda real-, y pudo costear estudios de pintura a su hijo en Roma, siguiendo con ello la tendencia de tantos pintores flamencos. El traslado de Silva a la romana via della Sirena, donde vivía entre 1634 y 1637, supondría un acercamiento a la embajada española situada en aquel barrio y al emba-

${ }^{36}$ J. de QUEVEDO, Historia del real monasterio de San Lorenzo, Madrid (Eusebio Aguado), 1854 (ed. Madrid, Hiperion, 1986), p. 175. 
jador, el portugués don Manuel de Moura marqués de Castelrodrigo, en un momento en que se estaban solicitando a Poussin, Lorena y varios pintores de origen flamenco abundante obra para el Buen Retiro. Quizá fue Moura quien le animó a marchar a España cuando terminó su embajada romana en 1641. Él mismo pediría al Rey que le allanara la entrada en el monasterio y palacio real del Escorial para que pudiera realizar copias de los cuadros que allí había, una lucrativa ocupación, como se demuestra por los 3.000 reales cobrados a Pötting por una copia de Tiziano, un precio que sería alto incluso para un original. Su tenencia de conserje del palacio, además de llegarle tardíamene por sus vínculos de amistad con los Herreras Barnuevos, no debía de llevar aparejado el cobro de gajes, y la ejercería por lo honorífico del servicio al rey y por las expectativas que creaba para su familia vinculándola al real sitio. Podría existir alguna dificultad cronológica para esta identificación que proponemos, pues su muerte en 1690 le otorgaría una avanzada edad incluso si nació hacia 1610, una fecha admisible para que habitara ya en Roma en 1630, si bien sería un poco arriesgado avanzar hasta 1615 . Pero no es imposible que muriera cerca de los ochenta años ocupando la tenencia de conserje, tanto más si su hijo, pintor como él, se hallaba a su lado, como parece.

\section{DOCUMENTOS}

№ 1. - 20-1-1660.

Poder de Diego de Silva para concertar la dote y arras de doña Clara de Vitoria y Riaño.

A.H.P.M., prot. 8.958, fol. 359-359v.

En la villa de Madrid a veinte días del mes de henero de mill y seiscientos y sesenta años, ante mi el escrivano y testigos pareció don Diego de Silva, maestro pintor del Sitio Real del Excurial, y dixo que tenía tratado y conferido de cassar y velarse con doña Mariana de Grijalva y Rriaño, hija lexítima de Eugenio Llorente de Grijalva y doña Clara de Vitoria y Rriaño, sus padres, y por quanto por las muchas obras y ocupaciones que tiene en el ministerio del dicho su oficio y ser considerables y de tanta ymportancia, no puede aussentar del dicho Rreal Sitio ni acudir para las diligencias y conciertos deste matrimonio, para lo qual otorga que da su poder cumplido, el que de derecho en tal casso se rrequiere y es necessario, a don Lorenço de Ssoto, assimismo maestro pintor, vezino desta dicha Villa, para que en su nombre rrepresentando su propia perssona pueda tratar, comunicar y concertar en la cantidad de la dotte que le han de dar con la dicha doña Mariana los dichos sus padres, y ofrecer las arras conforme a la calidad de su perssona, y hazer las capitulaciones con las condiciones y fuerças en derecho necessarias, y assimismo da poder general al dicho don Lorenço para que parezca ante el señor vicario general desta dicha Villa y saque los despacho o despachos que fuere necessario para el dicho casamiento, que desde luego se obliga de estar y pasar por lo que en virtud deste dicho poder hiziere y capitulase, representando su propia persona, que para lo susodicho se obliga con su perssona y vienes muebles y rayces y da poder a todas las justicias para que le compelan a ello por todo rigor de derecho, que el poder necesario ése le da para lo susodicho al dicho don Lorenço con todas yncidencias y dependencias, anexidades y conexidades, libre, franca y general administración, obligazión y relevazión y renunciaciones de leyes en forma, y así lo otorgó ante el escrivano, (tachado: y testigos), siendo testigos Luis de Salazar, Graviel de Castro y Balthasar de la Barrieta, residentes en esta Corte, y el otorgante, a quien yo, el escribano, doy fee conozco, firmó de su nombre. Don Diego de Silva. 


\section{№ 2. - 1669-1670.}

Memoriales de Sebastián de Herrera Barnuevo y de Diego de Silva solicitando la sucesión en la plaza de conserje del Escorial que tenía Manuel de Herrera Barmuevo, informe de la Junta de Obras y Bosques sobre los aspirantes y nombramiento.

A.G.P., Expediente personal de Sebastián de Herrera Barnuevo, 507/40.

Señor:

Don Sebastián de Herrera Barnuevo, maestro maior de las obras reales i ayuda de la furriera del Rey nuestro señor que Dios guarde, dice que por muerte de su hermano don Manuel de Herrera, a vacado el oficio de conserge del palacio real de San Laurencio, y porque dicho oficio le tenía asistiendo en Madrid a servir el oficio de ayuda de la furriera por no ser necesaria la asistencia personal en El Escorial, donde tenía una persona que cuidase del aseo de la calle asta llegar el casso de hacer jornada el Rey nuestro señor a dicho sitio, que sigue a su Magestad i no puede hacer falta; que es en la forma que le an tenido los más i que le tuvo Joachín Cobos, ayuda de la furriera.

Suplica a v. Mgd. le haga merced de dicho oficio en la forma que le tenía su hermano, atento a la descomodidad que le [ha] ocassionado su falta y las muchas obligaciones de su mucha familia, en que recivirá merced...

Pide el oficio de conserje del palacio del Escorial.

Señora:

Don Sebastián de Herrera Barnuevo, maestro mayor de V. Mgd.

La Reyna nuestra señora. 12 de diciembre 1669.

Remite un memorial de don Sebastián de Herrera en que pide el oficio de conserje del palacio de San Lorenço el Real que vacó por muerte de su hermano.

\section{Señora:}

Don Diego de Sylva, theniente de conçerje de San Lorenço el Real, postrado a los pies de v. Mgd. dice que su padre sirvió en el castillo de Amberes por pagador de vastimentos el espacio de 29 años, a cuya imitación el suplicante se dedicó también al real servicio, y con licencia de su $\mathrm{Mg}^{\mathrm{d}}$. el Rey (que está en el cielo) empleóse en el Escurial con el arte de la Pintura en copiar originales del Ticiano y otros, y assistió en aquel real sitio 26 años con su famlia, sirviendo estos dos postreros con el oficio de theniente de conçerje de aquella real casa, y exercitándolo con suma aprobación del revmo. Padre Prior y de todo el conbento, como constará a v. Mgd. por certificación de su revma. y sus oficios tan en favor y abono del suplicante y del proceder honrrado con que se ha portado no sólo en la puntual assistencia a las cosas de aquella real casa sino también con todos los moradores en dicho real sitio. Atento lo qual, vacando hoy día por muerte de don Manuel de Herrera el oficio de conçerje, llega a los reales pies de V. Mgd.

Suplicando a v. Mgd. muy humildemente que en consideración de los servicios de su padre y suyos, de los largos años que assiste en dicha real casa, y que se halla cargado con obligaciones de muger y cinco hixos, cuyo sustento, por su corta fortuna, pende de su cotidiano trabaxo, se sirba v. $\mathrm{Mg}^{\mathrm{d}}$. de honrrarle con la merced de dicho oficio, para que al passo que con este premio fuere mexorando de puesto y comodidades, cobre también nuebos alientos para continuar en el real serviçio como dessea hasta el último de su bida a ley de muy humildo y rendido criado de $\mathrm{v} . \mathrm{Mg}^{\mathrm{d}}$. 
D. Diego de Sylva, theniente de conçerje de San Lorenço el Real.

Pide el oficio de conserje del Escurial.

En el memorial incluso pretende don Diego de Silva, theniente de conserje del Palacio de San Lorenzo el Real se le haga maestro de la propiedad de este oficio que ha vacado por muerte de don Manuel de Herrera. Remítole a la junta de Obras y Bosques para que quando se me propusieren personas para él se cuyde de consultarle según sus servicios, partes y méritos.

En Madrid a 17 de diziembre de 669. A don Bernardino de Arando.

En Madrid a 2 de henero de 1670. Consúltensse para este oficio en primer lugar a don Sebastián de Herrera; en segundo a don Toribio González de los Corrales y en terzero a don Diego de Silba, por los méritos y serbicios que a cada uno le asisten.

(Al margen) Pressidente del Conssejo. Marqués del Fresno. Don Gerónimo de Camargo. Marqués de Salinas.

\section{Señora}

El oficio de conserje del Palacio y quarto de san Lorenço el real que servía don Manuel de Herrera ha vacado por su muerte. Tiene esta ocupación quatrocientos ducados de salario cada año, de los quales goça ciento de penssión doña María Cobos por los días de su vida.

Haviéndose visto en esta Junta los memoriales de los que pretenden que vuestra Magestad ha sido servida de remitir con decretos particulares y recordándose los servicios, méritos y partes de cada uno como v. $\mathrm{Mg}^{\mathrm{d}}$. manda ha parecido a la Junta proponer a v. Mgd. lo siguiente:

En primer lugar, a don Sevastián de Herrera, hermano del difunto, maestro mayor de las obras reales del Alcáçar desta
Villa y pintor de cámara del Rey nuestro señor, que Dios guarde, cuyos servicios son notorios.

En segundo a don Torivio Gonçález de los Corrales Caruz, rejidor de la ciudad de Obiedo, que pretendió este oficio en la ocasión que vacó por muerte de Manuel Cobos. Y ha servido no sólo en la guerra sino en todas las ocasiones de servicios que se han ofrecido hacer en la probincia y partido de Asturias, donde es natural, con muchas aprobación y celo del servicio de vuestra Magestad.

En tercer lugar a don Diego de Silba, que por estarle concedido al dicho don Manuel de Herrera pusiese perssona que acudiesse por él al exercicio de conserje, lo ha hecho y está haciendo al presente, ocupándose asimismo en lo que en el discursso deste tiempo se ha ofrecido en el exercicio de pintor, lo que ha sido requerido en aquel palacio real y combento.

V. Mgd. elijirá el que más fuere servida, con calidad que el que nombrare, no haviendo de residir en aquel sitio pueda poner en su lugar a su costa y por su quenta persona aprobada por esta Junta, que sirba por él. Madrid, 15 de henero de 1670.

Junta de Obras y Bosques. A 15 de henero de 1670

Propone personas para el officio de conserje del palacio, quarto de san Lorenço el Real que ha vacado por muerte de don Manuel de Herrera.

Nombro a don Sevastián de Herrera con la calidad que la Junta propone.

Publicada en 1 de hebrero 1670. Secretario don Bernardino de Arando. 